\title{
Cetoacidose Diabética Associada com Síndrome de Guillain-Barré: Relato de Caso
}

\section{apresentação de caso}

Thiago Bechara Noviello

TeResa CRISTINa B. Noviello

SAULO PURISCH

RODRIGO NUNes LAMOUNIER

JaNICE SEPÚlVEDa REIS

Patrícia A. F. da Cunha Menezes

Maria Regina Calsolari

Serviço de Endocrinologia e Metabologia da Santa Casa de Belo Horizonte, MG, Brasil.

Recebido em 31/07/2007 Aceito em 03/12/2007

\section{RESUMO}

A síndrome de Guillain-Barré (GBS) é uma desordem causada por exacerbada resposta imune aos processos infecciosos. O diabetes melito (DM) não é reconhecido como uma causa desta polirradiculopatia inflamatória, com poucos casos relatados na literatura sobre tal associação. Apresentamos um caso de uma paciente do sexo feminino, 44 anos, admitida com história recente de poliúria, polidipsia, perda de peso e astenia, glicemia de $562 \mathrm{mg} / \mathrm{dL}$, em cetoacidose, sem foco infeccioso. Posteriormente desenvolveu quadro de polirradiculopatia, insuficiência respiratória e alteração liquórica compondo o quadro de GBS. No presente relato, a paciente recuperou-se plenamente do déficit neurológico, assim como da hiperglicemia, configurando quadro de diabetes tipo 2, com tendência à cetoacidose, evoluindo sem insulino-dependência. (Arq Bras Endocrinol Metab 2008; 52/3:562-565)

Descritores: Síndrome de Guillain-Barré; Diabetes melito; Cetoacidose diabética

\section{ABSTRACT}

\section{Diabetes Ketoacidosis Associated with Guillan-Barré Syndrome.}

Guillain-Barré syndrome (GBS) is a disorder caused by exaggerated immune response to infectious process. Diabetes Melito (DM) is not recognized as one cause of this inflammatory polyradiculoneuropathy with just a few cases of this association been described in the literature so far. We report here the case of a 44 years-old female patient admitted with a history of polyuria, polydipsia, weight loss, asthenia, hyperglycemia $(562 \mathrm{mg} / \mathrm{dL}$ ) and ketoacidosis without any infectious focus. The patient progressed with poliradiculopathy, respiratory insufficiency and liquoric alteration completing the picture of Guillain-Barré syndrome. The patient fully recovered from the neurologic deficit and then stopped with insulin therapy. (Arq Bras Endocrinol Metab 2008; 52/3:562-565)

Keywords: Guillain-Barré syndrome; Diabetes mellitus; Diabetic ketoacidosis

\section{INTRODUÇÃO}

SÍNDROME DE GUILLAIN-BARRÉ (GBS) é uma polirradiculoneuropatia inflamatória autolimitada, na maioria das vezes do tipo desmielinizante, de mecanismo auto-imune, freqüentemente pós-infeccioso. Embora os mecanismos patogênicos ainda não estejam totalmente estabelecidos, tem-se mostrado que a resposta imune humoral e mediada por células contribui para a sua ocorrência. Em cerca de $20 \%$ a $50 \%$ dos casos podem ser detectados autoanticorpos anti-GMl (anti-glicofosfolipídeo l) e o processo pode ser desen- 
cadeado por infecção, sendo o agente infeccioso mais comumente relacionado como antecedente o Campylobacter jejuni. Em 20\% dos casos há história de diarréia precedendo o quadro (1). A doença geralmente tem evolução aguda (horas ou dias) sendo a manifestação mais comum a polirradiculoneuropatia inflamatória aguda desmielinizante (2). Geralmente apresenta quadro de fraqueza rapidamente progressiva, podendo evoluir para falência respiratória e com boa recuperação. Nos casos mais graves, pode-se instalar insuficiência respiratória, disautonomia, levando a arritmias cardíacas, sudorese e picos hipertensivos. Até $25 \%$ dos pacientes podem necessitar de ventilação mecânica, com mortalidade entre $4 \%$ e $10 \%$ dos casos, porquanto até $20 \%$ podem ficar com alguma seqüela (3). O diagnóstico é fundamentado nas características clínicas (fraqueza motora progressiva e arreflexia) e análise do liquor, que é o único critério laboratorial estabelecido. A elevação das proteínas e a presença de dez ou menos células mononucleares suportam o diagnóstico. A eletromiografia ajuda a diferenciar as características clínico-patológicas da doença $(2,4,5)$. O diabetes não é reconhecido como fator desencadeante da polirradiculopatia inflamatória, com poucos casos relatados na literatura sobre tal associação. Apresentamos um caso de cetoacidose diabética como primeira manifestação do diabetes com posterior evolução para GBS.

\section{RELATO DE CASO}

Paciente do sexo feminino, 44 anos, índice de massa corporal (IMC): 32, com história familiar negativa para DM ou doenças auto-imunes, admitida com história recente de poliúria, polidipsia, perda de peso e astenia, glicemia de $562 \mathrm{mg} / \mathrm{dl}$, com quadro clínico e laboratorial (Tabela 1 ) de cetoacidose diabética, sem foco infeccioso identificável. Dois dias após a internação e melhora da cetoacidose, a paciente iniciou quadro de fraqueza progressiva, apatia, dispnéia, sem alterações aos exames clínico e laboratoriais. Evoluiu em poucas horas com falência respiratória, tendo sido entubada e agravou-se com quadro de parada cardiorrespiratória, com boa resposta às manobras de reanimação, sendo transferida ao centro de terapia intensiva. Após 15 dias e múltiplas investigações, chegou-se ao diagnóstico de GBS, pela análise do liquor: proteínas $=294 \mathrm{mg} / \mathrm{dl}$; Pandy $(+++)$; células nucleadas $1 / \mathrm{mm}^{3}$ (Tabela 2 ), com tomografia computadorizada de crânio sem alterações significativas. Recebeu imunoglobulina, 27,5 g/dia, com recuperação da força muscular, mantendo-se insulinizada. Após alta hospitalar, com insulina NPH, foram dosados os marcadores imunológicos para diabetes tipo 1 , cujos resultados foram negativos, visto que o peptídeo $\mathrm{C}$ foi normal (Tabela 1). A dose de insulina foi reduzida e foi associada metformina, que não foi tolerada pela paciente. Ela des-

Tabela 1. Exames laboratoriais.

\begin{tabular}{lcc}
\hline Exames & Resultados & Valores de referência \\
\hline $\mathrm{pH}$ & 7,12 & $7,32 \mathrm{a} 7,43$ \\
\hline HCO3- & $7 \mathrm{mmoL} / \mathrm{L}$ & $22 \mathrm{a} 29 \mathrm{mmoL} / \mathrm{L}$ \\
\hline Cetonemia & +++ & negativo \\
\hline Glicemia & $562 \mathrm{mg} / \mathrm{dL}$ & $70 \mathrm{a} 99 \mathrm{mg} / \mathrm{dL}$ \\
\hline Leucócitos & $18.800 / \mathrm{mm}^{3}$ & $5.000 \mathrm{a} 10.000 / \mathrm{mm}^{3}$ \\
\hline Hemoglobina & $13,9 \mathrm{~g} / \mathrm{dL}$ & $14,4 \mathrm{a} 17,6 \mathrm{~g} / \mathrm{dL}$ \\
\hline Creatinina & $1,3 \mathrm{mg} / \mathrm{dL}$ & $0,8 \mathrm{a} 1,3 \mathrm{mg} / \mathrm{dL}$ \\
\hline Potássio & $3,2 \mathrm{mEq} / \mathrm{L}$ & $3,7 \mathrm{a} 5,6 \mathrm{mEq} / \mathrm{L}$ \\
\hline Sódio & $141 \mathrm{mEq} / \mathrm{L}$ & $135 \mathrm{a} 145 \mathrm{mEq} / \mathrm{L}$ \\
\hline Uréia & $22 \mathrm{mg} / \mathrm{dL}$ & $10 \mathrm{a} 40 \mathrm{mg} / \mathrm{dL}$ \\
\hline Anti-GAD & $0,3 \mathrm{U} / \mathrm{mL}$ & $<1,0 \mathrm{U} / \mathrm{mL}$ \\
\hline Anti-IA2 & $0,4 \mathrm{U} / \mathrm{mL}$ & $<0,5 \mathrm{U} / \mathrm{mL}$ \\
\hline Peptídeo C & $4,3 \mathrm{ng} / \mathrm{dL}$ & $1,1 \mathrm{a} 5,0 \mathrm{ng} / \mathrm{dL}$ \\
\hline
\end{tabular}


Tabela 2. Análise do liquor.

\begin{tabular}{|c|c|c|}
\hline Exames & Resultados & Valores de referência \\
\hline Proteínas & 294 mg/dl pandy +++ & 15 a $45 \mathrm{mg} / \mathrm{dL}$ \\
\hline Glicemia & $122 \mathrm{mg} / \mathrm{dL}$ & 2/3 a glicemia sérica \\
\hline Citometria & $\begin{array}{c}1 \mathrm{cel} \text {. nucleada } / \mathrm{mm}^{3} \\
5 \mathrm{~h} \mathrm{em} / \mathrm{mm}^{3}\end{array}$ & $\begin{array}{c}\text { 0-5 mononucleadas } / \mathrm{mm}^{3} \\
\text { Ausência de hemácias } \\
\text { Ausência de leucócitos PMN }\end{array}$ \\
\hline Baterioscopia pelo Gram & Não foram vistas bactérias & Ausência de bactérias \\
\hline
\end{tabular}

continuou o acompanhamento no serviço, retornando à sua cidade de origem, fazendo seguimento com baixas doses de insulina NPH (04 UI/dia), assintomática, com relato de controle glicêmico satisfatório.

\section{DISCUSSÃo}

A GBS é uma polirradiculopatia inflamatória aguda autolimitada. O seu tratamento é fundamentado no suporte clínico e no uso da imunoglobulina intravenosa $(0,4 \mathrm{~g} / \mathrm{kg}$ de peso por cinco dias), como o realizado para a paciente descrita. Isso é suportado pela ação da imunoglobulina por meio de mecanismos imunológicos e pelos resultados clínicos descritos (6).

A GBS geralmente é precedida por um fator causal. Entre eles estão os infecciosos, tendo como o principal agente o Campylobacter jejuni, podendo também ocorrer após vacinas, cirurgias, transplante renal, lúpus eritomatoso sistêmico, sarcoidose, linfoma e até por picada de cobra (1). O diabetes tem sido associado com GBS em poucos casos descritos até o momento. Em 1990, Flax relatou quatro casos, nos quais foi verificada resistência à insulina durante a manifestação aguda da GBS, mostrando uma complicação metabólica da doença, haja vista que até então a GBS não era reconhecida como causa de resistência insulínica. Dos quatro pacientes, dois precisaram usar insulina durante breve período (7). Em 2000, foi relatado um caso de uma mulher, 37 anos, admitida em cetoacidose diabética, com anticorpo negativo. No quinto dia de internação após recuperação da cetoacidose, a paciente apresentou quadro de fraqueza motora, insuficiência respiratória e após propedêutica foi confirmado o diagnóstico de GBS. Essa paciente apresentou disfunção autonômica e hipotensão postural persistente após recuperação do quadro, mostrando uma evolução atípica da
GBS (8). Em 1992, Foster e Long mostraram a associação de GBS, cetoacidose diabética e pneumonia. GBS se apresentou logo após a recuperação da cetoacidose (9). Marie Rouanet-Larriviere publicou uma descrição com dois casos de polirradiculopatia inflamatória desmielinizante após quadro de cetoacidose diabética, em que foi feito o diagnóstico de GBS por meio da biópsia do nervo periférico, mostrando a importância do diagnóstico diferencial com neuropatia diabética aguda, que geralmente é dolorosa em paciente com quadro de caquexia (10).

No caso relatado, a paciente apresentou quadro de cetoacidose diabética clássica, com posterior manifestação de fraqueza periférica e insuficiência respiratória aguda, apresentando exame do liquor típico, evidenciando a hipótese de GBS, sendo tratado com imunoglobulina e suporte clínico, com posterior melhora. O uso de imunoglobulina é bem estabelecido na literatura, mostrando-se tão eficiente quanto a plasmaférese (11). Já o uso de corticosteróide tem eficácia discutível, visto que pode aumentar o risco de diabetes melito (12).

Considerando-se o diagnóstico de diabetes melito tipo 1 (DMl), sabe-se que as duas moléstias (DMl e GBS) apresentam mecanismos auto-imunes envolvidos nos processos de fisiopatologia, levantando a hipótese de anticorpos envolvidos em tais processos, embora esses mecanismos sejam ainda pouco conhecidos. Um aspecto interessante neste caso é que a paciente apresentou quadro clínico sugestivo de DMl (cetoacidose sem evidência de infecção), porém o quadro laboratorial sugeria o diagnóstico de diabetes melito tipo 2 (DM2) (auto-anticorpos negativos e peptídeo C preservado). A associação de cetoacidose e diagnóstico de DMl classicamente proposta tem sido questionada como preditor de insulinização definitiva, principalmente em pacientes adultos. Se por um lado, entre pa- 
cientes com DM2, aqueles que apresentam CAD tendem a ter peptídeo C significativamente menor que pacientes com DM2 sem essa tendência (13), por outro, a diferenciação diante de um quadro de CAD entre DM2 e DMl pode, muitas vezes ser difícil. Diversos esquemas tem sido propostos, desde o classicamente descrito pela American Diabetes Association, em que pacientes com CAD têm DMl, sendo considerados idiopáticos aqueles sem marcadores sorológicos de auto-imunidade contra as ilhotas pancreáticas, até outros que propõem o índice de massa corporal, a presença ou não de anticorpos, a avaliação da função da célula-beta e, finalmente o critério combinado, que correlaciona a auto-imunidade com os níveis de peptídeo $\mathrm{C}$ em jejum. De acordo com Balasubramanyam e cols. este último seria o critério de melhor acurácia na predição de insulino-dependência no longo prazo (13).

Neste caso relatado, a paciente apresenta quadro de DM2, cuja manifestação inicial se deu com CAD, evoluindo com GBS e posterior recuperação, tanto neurológica quanto da homeostase glicêmica, à semelhança dos casos descritos por Flax, em 1990, em que a maioria dos pacientes fez uso apenas temporário de insulina e a característica dominante da hiperglicemia relacionada a GBS foi a resistência insulínica.

Ainda é desconhecido o fator desencadeante comum entre o diabetes manifesto e a GBS. Em 1998, Niklason e cols., observando a concentração de casos novos de GBS, DMl e a evolução da população de roedores, com picos a cada quatro a cinco anos na Suécia, propôs a hipótese de um agente infeccioso comum, que explicasse a sazonalidade (14). Algo, entretanto, ainda inconsistente e distante diante de um caso de cetoacidose em paciente com DM2, sem evidências de auto-imunidade evoluindo com essa polirradiculopatia inflamatória.

\section{REFERÊNCIAS}

1. Seneviratne U. Guillain-Barré syndrome. Posgrad Med J. 2000;76:774-82.

2. Rees JH, Soudian SE, Gregon NA, et al. Campylobacter jejuni infections and Guillian-Barré syndrome. N Engl J Med. 1995;333:1374-9.

3. Hughes RAC, Cornblath DR. Guillain-Barré syndrome. Lancet. 2005;366:1653-66

4. McKhann GM, Cornblath DR, Griffin JW, et al. Acute motor axonal neuropathy: a frequent cause of acute flaccid paralysis in China. Ann Neurol. 1993;33:333-42.

5. Asbury AK, Cornblath DR. Assessment of current diagnostic criteria for Guillain-Barré syndrome. Ann Neurol 1990;27 Suppl:S21-4.

6. Kleyweg RP, van der Meche FGA, Meultee J. Treatment of Guillain-Barré syndrome with high-dose gammaglobulin. Neurology. 1988;38:1639-41.

7. Flax H, Matthews DR. Diabetes associated with Guillain-Barré syndrome. Diabetes Res. 1990;14(1):47-50.

8. Fujiwara S, Oshika H, Motoki K. Diabetes Ketoacidosis Associated with Guillain-Barré syndrome with Autonomic Dysfunction. Intern Med. 2000;39(6):495-8.

9. Marie Rouanet-larriviere, Claude Vital, Pierre Arne. GuillainBarré syndrome occurring in two women after ketoacidosis comatose state disclosing an insulin-dependent diabetes mellitus. J Peripher Nerv Syst. 2000;5:27-31.

10. Hughes RAC, Raphael J-C, Swan AV, van Doorn PA. Intravenous immunoglobulin for Guillain-Barré syndrome (cochrane review). In: The Cochrane Database of Systematic Reviews, 1. Chichester, UK: John Wiley and Sons, Ltd., 2006.

11. Hughes RA, Swan AV, van Koningsveld R, van Doorn PA. Corticosteroids for Guillain-Barre syndrome. Cochrane Database Syst Rev. Chichester, UK: John Wiley and Sons, Ltd., 2006.

12. Linfoot $P$, Bergstrom $C$, Ipp E. Pathophysiology of ketoacidosis in type 2 diabetes mellitus. Diabet Med. 2005;22:1414-9.

13. Balasubramanyam $A$, et al. Accuracy and predictive value of classification schemes for ketosis prone diabetes. Diabetes Care. 2006;29:2575-9.

14. Niklason B, Hornfeldt B, Lundrran B. Could myocarditis, insulin-dependent diabetes mellitus, and Guillain-Barré syndrome be caused by one or more infectious agents carried by rodents? Emerg Infect Dis. 1998;4:187-93.

Endereço para correspondência:

Thiago Bechara Noviello

CEPCEM

Av. Francisco Sales, $1111,5^{\circ}$ andar, ala D - Santa Efigênia

30150-221, Belo Horizonte MG

E-mail: thiagobn5@hotmail.com 